\title{
离子液体水溶液 $\mathrm{pH}$ 值测定及酸碱特性研究
}

\author{
杨翠莲皇甫立霞粟航郭开华*
}

(中山大学工学院 广州 510006)

\begin{abstract}
摘要 在离子液体水溶液作为新一代优良吸收工质的工业应用研究和开发中, 对该体系的物理化学性质(诸如离子液 体在水中存在性状及其酸碱性问题等)的深入认识和掌握尤为重要. 本文采用 $\mathrm{pH}$ 酸度计和精密 $\mathrm{pH}$ 试纸两种方法, 对水 质量分数分别为 $0.04 \sim 0.95$ 的 1 -乙基-3-甲基咪唑醋酸盐([EMIM]Ac)和 $0.01 \sim 0.96$ 的 1-已基-3-甲基咪唑氯盐 ([HMIM]Cl)离子液体水溶液的 $\mathrm{pH}$ 值进行测试和定量分析. 测试结果表明 $\mathrm{pH}$ 酸度计和精密 $\mathrm{pH}$ 试纸两种方法的测试结 果有较大的差异, 这种差异是由于离子液体一水混合体系中局部形态与整体特性不同造成的. 本文成功拟合了 $\mathrm{pH}$ 精密 试纸测定值，计算获得的这两种离子液体水溶液的酸浓度与测定值基本符合，精密试纸测定的 $\mathrm{pH}$ 值可用于表征离子液 体水溶液整体酸碱特性. 基于离子液体在水溶液中形成惰化聚合膜的假设, 提出了分别表征聚合膜水溶解度和离子液 体水溶液水活度系数的溶液理论模型, 根据溶液相平衡理论建立了聚合膜中氢离子浓度与水溶液中氢离子浓度差别的 计算方法, 成功拟合了 $\mathrm{pH}$ 酸度计测定值. 研究结果为离子液体水溶液体系的 $\mathrm{pH}$ 值和酸碱度分析提供了实验依据和预 测理论模型.
\end{abstract}

关键词 离子液体; 水溶液; $\mathrm{pH}$ 值; 酸碱性

\section{Determination of $\mathrm{pH}$ Value and Acid-base Property of Ionic Liquid Aqueous Solutions}

\author{
Yang, Cuilian Huangfu, Lixia Su, Hang Guo, Kaihua* \\ (Sun Yat-Sen University, School of Engineering, Guangzhou 510006)
}

\begin{abstract}
The ionic liquids (ILs) and their aqueous solutions have been considered as a new generation of absorption working substances for applications in gas separation and absorption refrigeration processes. It is important to understand the physicochemical properties of ionic liquids in aqueous solutions, such as chemical potential and acid-base properties before any industry application. In this study, we focused on the acid-base properties of the IL aqueous solutions by testing the $\mathrm{pH}$ values of the aqueous solutions of two ionic liquids, 1-ethyl-3-methylimidazolium acetate ([EMIM]Ac) and 1-hexyl-3-methylimidazolium chloride $([\mathrm{HMIM}] \mathrm{Cl})$. All the tests were carried out at $25{ }^{\circ} \mathrm{C}$ and in a well controlled dry air surrounding. There were 16 water mass fractions for each of [EMIM]Ac and [HMIM] Cl aqueous solutions tested in range of 0.04 to 0.95 and 0.01 to 0.96 , respectively. There, two methods, the sophisticated $\mathrm{pH}$ paper and the $\mathrm{pH}$ meter, were applied for the tests and the differences between the two test methods were detected. The test data from the two test methods and their differences were then evaluated with an aggregation film model and the activity theory for the film and aqueous solution phases. The results show that the difference of the $\mathrm{pH}$ values tested by the two methods is significant. The $\mathrm{pH}$ data from the sophisticated $\mathrm{pH}$ paper test present the bulk acid-base property of the IL aqueous solutions, while the $\mathrm{pH}$ meter can only reflect the hydrogen ion concentration in the IL aggregation film which was formed on the surface of the electrode of the $\mathrm{pH}$ meter. With analysis of acid-base reaction in the IL aqueous solution, a calculation model for the $\mathrm{pH}$ value of the bulk solution region was developed, and the data of the sophisticated $\mathrm{pH}$ paper test were correlated and the solution acid-base values were obtained. Based on analysis of the water species equilibrium in the IL aggregation film and the bulk aqueous solution phases and its effect on the concentration of hydrogen ions in the aggregation film, a method for calculation of the difference of $\mathrm{pH}$ values between the aggregation film and the bulk solution was proposed and the data from the $\mathrm{pH}$ meter were then fitted with the proposed calculation method. The results provide experimental data and a quantitative method for analysis of the acid-base property of IL aqueous solution systems.
\end{abstract}

Keywords ionic liquid; aqueous solution; $\mathrm{pH}$ value; acid-base property

*E-mail: Guokh120@163.com; Tel.: 020-39332893

Received September 29, 2013; published January 16, 2014.

Supporting information for this article is available free of charge via the Internet at http://sioc-journal.cn.

Project supported by the National Natural Science Foundation of China (No. 51076169) and the Natural Science Foundation of Guangdong Province (No. 9251027501000001).

项目受国家自然科学基金(No. 51076169)和广东省自然科学基金(No. 9251027501000001)重点项目资助. 


\section{1 引言}

随着国家对绿色清洁能源的日益重视, 离子液体 (Room Temperature Ionic Liquids, RTILs)作为功能材料 和新型介质的理论研究和开发应用备受人们的关注 ${ }^{[1 \sim 4]}$. 水溶性离子液体除满足吸收式循环工质的基本要求, 还 因其蒸汽压几乎为零、室温下可流动等特性克服了目前 氨一水和溴化锂一水制冷系统存在的诸如有毒、可燃、结 晶、腐蚀性强等缺点, 已成为热洜和制冷行业的研究热 点之一 ${ }^{[5,6]}$. 目前, 关于离子液体的研究主要集中在离子 液体的热物性, 如比热特性 ${ }^{[7,8]}$ 、密度特性 ${ }^{[9]}$ 、蒸汽压特 性 ${ }^{[10,11]}$ 、吸收特性 ${ }^{[12,13]}$ 、对有机物溶解特性 ${ }^{[14]}$ 、热稳定 性 ${ }^{[15]}$ 等.

离子液体在物理化学转换过程中大部分受环境酸 碱度影响. Aggarwal 等 ${ }^{[16]}$ 发现在碱性条件下咪唑阳离子 会与 Michael 加成反应的反应物发生反应, 咪唑型离子 液体不适合碱催化反应. Seddon 等 ${ }^{[17]}$ 研究发现由于阴离 子的结构决定了含卤离子液体的酸碱性, 并曾提出用卤 离子浓度的负对数定量描述这类离子液体的酸碱性, 但 随着离子液体种类的增多, 离子液体的阴离子不再局限 于卤元素, 这种定义酸碱度的方法难以得到推广. Zhao 等 ${ }^{\left[{ }^{[18]}\right.}$ 应用电位滴定的方法, 用氢氧化钠溶液作为标准 滴定液, 测得了一系列 1,2 取代基含有羧基的咪唑卤盐 离子液体的解离平衡常数. Thomazeau 等 ${ }^{[19]}$ 研究非氯代 铝酸盐离子液体的酸碱特性时, 提出使用哈密特方程进 行表征. Yang 等 ${ }^{[20]}$ 用分子探针-红外光谱分析法, 根据 吡啶分子探针的骨架震动吸收峰的特性, 定性测定了 $\mathrm{Cl}^{-}$为阴离子的室温离子液体的 Lewis 和 Bronsted 酸性. 陈学伟等 ${ }^{[21]}$ 应用电位滴定法研究了碱性离子液体的 $p K_{\mathrm{b}}$ 值. 离子液体的酸碱特性的研究和确定对于离子液体的 工程应用有非常重要的意义.

离子液体水溶液体系在吸收和分离等化工过程以 及制冷和热泵等能源利用过程中的应用中, 人们关注的 焦点之一是其酸碱特性. 测量酸碱性最简单、直观的方 法是使用 $\mathrm{pH}$ 酸度计或 $\mathrm{pH}$ 试纸. 然而目前仅有 Ober 等 ${ }^{[22]}$ 用 $\mathrm{pH}$ 酸度计对 $0 \sim 25 \%$ 的 1-乙基-3-甲基咪唑醋酸 盐水溶液的 $\mathrm{pH}$ 值进行了测定, 这方面研究工作相对缺 乏. 在化工和能源工程领域, 一些亲水性咪唑型离子液 体的研究受到重视, 特别是 1-乙基-3-甲基咪唑醋酸盐 ([EMIM]Ac)和 1-己基-3-甲基咪唑氯盐([HMIM]Cl), 由 于其热稳定性高 (分解温度分别为 222.6 和 $264.8{ }^{\circ} \mathrm{C}$ ), 而 且与水可充分互溶, 非常有可能在气体吸收、萃取分离 和吸收式制冷等化学和能源工程中获得应用 ${ }^{[23]}$. 在吸 收式制冷和热百循环中, 由于这两种离子液体的强吸湿 性和水溶液的优良蒸汽压特性, 有可能成为优良的吸收 式制冷和热百的工质 ${ }^{[24]}$. 这些应用都对 [EMIM]Ac 和 $[\mathrm{HMIM}] \mathrm{Cl}$ 离子液体水溶液的酸碱特性研究提出了需 求.

本文采用 $\mathrm{pH}$ 酸度计和精密 $\mathrm{pH}$ 试纸两种方法, 对水
的质量分数为 $0.04 \sim 0.95$ 的 [EMIM]Ac 和 $0.01 \sim 0.96$ 的 $[\mathrm{HMIM}] \mathrm{Cl}$ 水溶液 $\mathrm{pH}$ 值进行测定和定量分析, 从而了解 离子液体-水混合体系的 $\mathrm{pH}$ 值及酸碱度随组分份额的 变化规律, 建立相应的计算模型并完善这种混合体系的 溶液理论模型.

\section{2 结果与讨论}

\section{1 实验结果}

用 $\mathrm{pH}$ 酸度计和精密 $\mathrm{pH}$ 试纸测得不同浓度的 $[\mathrm{EMIM}] \mathrm{Ac}$ 和 $[\mathrm{HMIM}] \mathrm{Cl}$ 水溶液 $\mathrm{pH}$ 值分别列于表 1 和表 2. 表中 $p H_{\text {exp }}$ 为 $\mathrm{pH}$ 测定值, $w_{1}$ 为测试样品水的质量分 数. [EMIM] Ac 和 $[\mathrm{HMIM}] \mathrm{Cl}$ 两种离子液体水溶液的 $p H_{\text {exp }}$ 随溶液浓度的变化关系分别示于图 2 和图 3, 由图 中散点示出.

由图 2 和图 3 可见, 当水质量分数较大时 ([EMIM]Ac 溶液 $w_{1}>0.65$ 和 [HMIM]Cl 溶液 $w_{1}>0.44$ ), 两种方法测得的 $\mathrm{pH}$ 值基本一致, 分别在 7.10 7.80 和 7.40 7.90 范围内; 而当水质量分数较小时, 两种方法 测得的离子液体水溶液的 $\mathrm{pH}$ 值有显著的差异. 随着水 的份额不断减少, $\mathrm{pH}$ 酸度计测得的 $\mathrm{pH}$ 值逐渐偏离试纸 测得的 $\mathrm{pH}$ 值. $\mathrm{pH}$ 酸度计测得的 [EMIM] Ac 水溶液 $\mathrm{pH}$ 值 随水含量减少而增加的非常显著, 当 $w_{1}=0.038$ 时测得 的 $p H_{\mathrm{exp}}=14.15$, 与试纸测得的 $\mathrm{pH}$ 值偏离大于 7. 对于 $[\mathrm{HMIM}] \mathrm{Cl}$ 水溶液, $\mathrm{pH}$ 值偏离略小, 当 $w_{1}=0.01$ 时, 酸 度计测得的 $p H_{\mathrm{exp}}=7.34$, 与试纸测得的 $\mathrm{pH}$ 值偏离大于 1. 显然 $\mathrm{pH}$ 酸度计测得的并非实际水溶液的整体 $\mathrm{pH}$ 值, 而是靠近测量电极球泡壁面处的局部氢离子浓度 $(\mathrm{pH}$ 值), 不能表征整体离子液体水溶液的酸碱特性. 这种 现象可由下一节的分析解释, 并可根据理论分析获得定 量计算模型.

\section{2 理论分析}

对于离子液体浓度较大时两种方法的测定结果有 较大差异, 本文认为其原因是: 对于精密 $\mathrm{pH}$ 试纸测定 方式，由于受溶液本身黏性、扩散特性、内部微观形态 等性质影响较小, 反映的是溶液的总体酸碱特性; 而对 于 $\mathrm{pH}$ 酸度计测定方法, 由于在离子液体浓度较大时离 子液体在其水溶液中的缔合惰化作用 ${ }^{[25 ~ 27]}$, 形成的缔 合离子液体分子团包裹着 $\mathrm{pH}$ 酸度计的测量电极, 造成 水分子扩散困难, $\mathrm{pH}$ 酸度计只能测到其周围离子液体 聚合膜中溶解的少量氢离子, 造成 $\mathrm{pH}$ 值偏高, 测定结 果不具有整体性.

这种离子液体缔合作用造成测量电极外壁形成离 子聚合层现象可由图 1 所示物理模型表示. 假设在 $\mathrm{pH}$ 酸度计测量电极外壁(A 相)外表面形成一个离子液体组 成的聚合膜( $\mathrm{B}$ 相), 该聚合膜由惰化和非惰化两类离子 液体形态构成, 惰化形态对水无溶合作用而非惰化形态 对水有一定的吸收能力, 可由亨利系数来表征. C 相表 
表 1 [EMIM]Ac 水溶液 $\mathrm{pH}$ 实验值与计算值

Table $1 \mathrm{pH}$ Value experimental and calculated data for [EMIM]Ac aqueous solution

\begin{tabular}{|c|c|c|c|c|c|c|}
\hline \multirow{2}{*}{$\begin{array}{l}\text { Water mass fraction } \\
\qquad w_{1}(w / w)\end{array}$} & \multicolumn{3}{|c|}{$\mathrm{pH}$ Meter experimental and calculated values } & \multicolumn{3}{|c|}{ Sophisticated $\mathrm{pH}$ paper experimental and calculated values } \\
\hline & $p H_{\text {exp }}$ & $p H_{\text {cal }}^{\prime}$ & $\mathrm{RD}$ & $p H_{\text {exp }}$ & $p H_{\text {cal }}$ & $\mathrm{RD}$ \\
\hline 0.038 & 14.15 & 13.30 & $-5.99 \%$ & 6.80 & 6.90 & $1.49 \%$ \\
\hline 0.066 & 13.11 & 12.96 & $-1.19 \%$ & 6.90 & 6.97 & $1.01 \%$ \\
\hline 0.097 & 12.60 & 12.58 & $-0.12 \%$ & 6.90 & 7.04 & $2.07 \%$ \\
\hline 0.137 & 11.42 & 12.13 & $6.23 \%$ & 7.10 & 7.13 & $0.37 \%$ \\
\hline 0.198 & 11.31 & 11.48 & $1.55 \%$ & 7.10 & 7.24 & $1.95 \%$ \\
\hline 0.297 & 10.12 & 10.53 & $4.10 \%$ & 7.20 & 7.38 & $2.47 \%$ \\
\hline 0.488 & 8.85 & 9.07 & $2.42 \%$ & 7.50 & 7.51 & $0.11 \%$ \\
\hline 0.500 & 8.51 & 8.99 & $5.64 \%$ & 7.60 & 7.51 & $-1.17 \%$ \\
\hline 0.550 & 8.26 & 8.68 & $5.07 \%$ & 7.60 & 7.52 & $-1.12 \%$ \\
\hline 0.600 & 8.01 & 8.40 & $4.85 \%$ & 7.60 & 7.51 & $-1.21 \%$ \\
\hline 0.650 & 7.82 & 8.15 & $4.23 \%$ & 7.60 & 7.49 & $-1.44 \%$ \\
\hline 0.700 & 7.65 & 7.93 & $3.61 \%$ & 7.50 & 7.46 & $-0.49 \%$ \\
\hline 0.750 & 7.51 & 7.73 & $2.90 \%$ & 7.50 & 7.43 & $-0.99 \%$ \\
\hline 0.800 & 7.39 & 7.56 & $2.30 \%$ & 7.50 & 7.38 & $-1.62 \%$ \\
\hline 0.850 & 7.29 & 7.41 & $1.60 \%$ & 7.30 & 7.32 & $0.31 \%$ \\
\hline 0.900 & 7.21 & 7.28 & $1.02 \%$ & 7.10 & 7.26 & $2.21 \%$ \\
\hline 0.950 & 7.14 & 7.18 & $0.53 \%$ & 7.20 & 7.18 & $-0.24 \%$ \\
\hline
\end{tabular}

表 $2[\mathrm{HMIM}] \mathrm{Cl}$ 水溶液 $\mathrm{pH}$ 实验值与计算值

Table $2 \mathrm{pH}$ Value experimental and calculated data for [HMIM]Cl aqueous solution

\begin{tabular}{|c|c|c|c|c|c|c|}
\hline \multirow{2}{*}{$\begin{array}{l}\text { Water mass fraction } \\
\qquad w_{1}(w / w)\end{array}$} & \multicolumn{3}{|c|}{$\mathrm{pH}$ Meter experimental and calculated values } & \multicolumn{3}{|c|}{ Sophisticated $\mathrm{pH}$ paper experimental and calculated values } \\
\hline & $p H_{\exp }$ & $p H_{\text {cal }}{ }^{\prime}$ & $\mathrm{RD}$ & $p H_{\exp }$ & $p H_{\text {cal }}$ & $\mathrm{RD}$ \\
\hline 0.010 & 7.34 & 7.39 & $0.62 \%$ & 6.15 & 6.18 & $0.41 \%$ \\
\hline 0.021 & 7.37 & 7.39 & $0.19 \%$ & 6.30 & 6.21 & $-1.53 \%$ \\
\hline 0.036 & 7.35 & 7.39 & $0.49 \%$ & 6.15 & 6.24 & $1.48 \%$ \\
\hline 0.054 & 7.34 & 7.39 & $0.69 \%$ & 6.30 & 6.29 & $-0.19 \%$ \\
\hline 0.079 & 7.35 & 7.40 & $0.70 \%$ & 6.30 & 6.35 & $0.77 \%$ \\
\hline 0.115 & 7.34 & 7.40 & $0.80 \%$ & 6.30 & 6.44 & $2.11 \%$ \\
\hline 0.173 & 7.36 & 7.42 & $0.82 \%$ & 6.30 & 6.57 & $4.09 \%$ \\
\hline 0.258 & 7.36 & 7.44 & $1.09 \%$ & 6.75 & 6.75 & $0.07 \%$ \\
\hline 0.444 & 7.41 & 7.50 & $1.27 \%$ & 6.90 & 7.11 & $2.95 \%$ \\
\hline 0.500 & 7.50 & 7.52 & $0.32 \%$ & 7.35 & 7.20 & $-2.03 \%$ \\
\hline 0.600 & 7.62 & 7.57 & $-0.63 \%$ & 7.50 & 7.36 & $-1.91 \%$ \\
\hline 0.700 & 7.70 & 7.62 & $-1.03 \%$ & 7.80 & 7.50 & $-4.06 \%$ \\
\hline 0.800 & 7.77 & 7.67 & $-1.30 \%$ & 7.50 & 7.61 & $1.51 \%$ \\
\hline 0.900 & 7.78 & 7.73 & $-0.54 \%$ & 7.80 & 7.72 & $-1.09 \%$ \\
\hline 0.930 & 7.96 & 7.75 & $-2.62 \%$ & 7.65 & 7.74 & $1.20 \%$ \\
\hline 0.961 & 7.95 & 7.77 & $-2.16 \%$ & 7.80 & 7.77 & $-0.40 \%$ \\
\hline
\end{tabular}

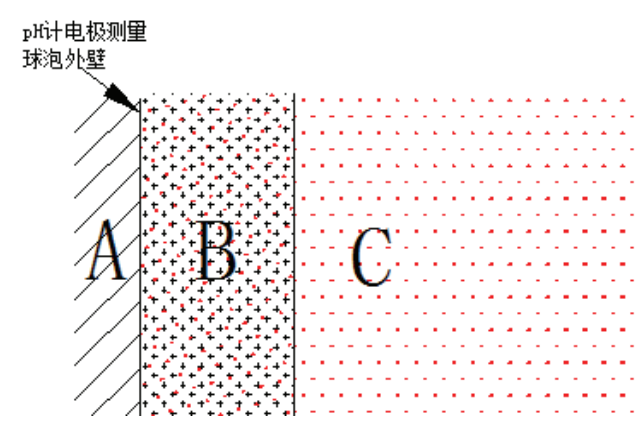

图 $1 \mathrm{pH}$ 计外壁惰化和非惰化离子液体水溶液分层模型图

Figure 1 Schematic diagram of $\mathrm{pH}$ meter

(A) Measuring electrode of $\mathrm{pH}$ meter; (B) polymer film forming by idle (black dots) and non-idle (red dots) ionic liquids; (C) non-idle ionic liquids aqueous solution
示存在于聚合膜外部的离子液体水溶液区域，占整个离 子液体一水体系的绝大部分, 其中离子液体为非惰化形 态，与水相互作用形成溶液体系，可由溶液理论来描述. 以下分别对外部溶液体系(代表整体混合体系，可由试 纸测得的 $\mathrm{pH}$ 值来表征)和聚合膜体系(代表近壁面极小 非完全溶液体系，由酸度计测得的 $\mathrm{pH}$ 值来表征)的酸碱 特性和氢离子浓度建立计算模型.

\subsection{1 离子液体水溶液氢离子浓度计算模型}

离子液体水溶液的酸碱度是水和离子液体共同作 用的结果. 假设离子液体溶液中，离子液体的阴离子与 水相互作用产生的酸(HD)发生如下解离平衡:

$$
\mathrm{HD} \rightleftharpoons \mathrm{H}^{+}+\mathrm{D}^{-}
$$

该解离反应的平衡常数可由下式表达: 


$$
K_{\mathrm{a}}=\frac{C\left(\mathrm{H}^{+}\right) C\left(\mathrm{D}^{-}\right)}{C(\mathrm{HD})}
$$

式中 $K_{\mathrm{a}}$ 为酸 $(\mathrm{HD})$ 的解离平衡常数. 假设由离子液体作 为溶质造成溶剂(水)酸碱不平衡而产生的酸浓度为 $C_{1}$, 对应的氢离子浓度为 $C\left(\mathrm{H}^{+}\right)_{1}$; 同理, 假设由水造成溶剂 (离子液体)酸碱不平衡而产生的酸浓度为 $C_{2}$, 对应的氢 离子浓度为 $C\left(\mathrm{H}^{+}\right)_{2}$. 可认为溶液中 $C\left(\mathrm{H}^{+}\right)=C\left(\mathrm{D}^{-}\right)$, 则 由式(2)可得 $C\left(\mathrm{H}^{+}\right)_{1}$ 和 $C\left(\mathrm{H}^{+}\right)_{2}$ 分别为:

$$
\begin{aligned}
& C\left(\mathrm{H}^{+}\right)_{1}=\sqrt{C_{1} K_{\mathrm{a} 1}} \\
& C\left(\mathrm{H}^{+}\right)_{2}=\sqrt{C_{2} K_{\mathrm{a} 2}}
\end{aligned}
$$

因为离子液体在水溶液中缔合形成分子团, 其分子 量无法准确确定, 所以用体积分数来表征各组分的作用 能力. 水溶液中水和离子液体体积分数 $\varphi_{1}$ 和 $\varphi_{2}$ 可由质 量分数 $w_{1}$ 和 $w_{2}$ 表示:

$$
\begin{aligned}
& \varphi_{1}=\frac{w_{1} \rho_{2}}{w_{1} \rho_{2}+w_{2} \rho_{1}} \\
& \varphi_{2}=\frac{w_{2} \rho_{1}}{w_{1} \rho_{2}+w_{2} \rho_{1}}
\end{aligned}
$$

根据稀溶液理论, 若在水中加入极少量离子液体或 在离子液体中加入极少量水形成的稀溶液造成溶剂酸 碱不平衡产生的酸浓度分别为 $C_{10}$ 和 $C_{20}$, 对应的 $\mathrm{pH}$ 值 为 $p H_{10}$ 和 $p H_{20}$. 假设在一定溶液组分下溶质容积份额 对体系中酸浓度的作用可由幂指数关系表达, 则 $C_{1}$ 和 $C_{2}$ 可由式(7)和(8)表示:

$$
\begin{aligned}
& C_{1}=C_{10} A_{2,1}^{\varphi_{2}} \\
& C_{2}=C_{20} A_{1,2}{ }^{\varphi_{1}}
\end{aligned}
$$

式中 $A_{2,1}$ 和 $A_{1,2}$ 为常数, 分别表征离子液体 2 对水 1 和 水 1 对离子液体 2 的作用系数. 上两式满足当溶质容积 分数 $\varphi_{1}$ 和 $\varphi_{2}$ 分别趋于零时 $C_{1}=C_{10}$ 和 $C_{2}=C_{20}$ 的边界条 件.

离子液体与水在溶液体系中共同作用产生的总的 氢离子浓度 $C\left(\mathrm{H}^{+}\right)$可由适当设定的混合规则来表示. 这 里根据氢离子浓度由 $\mathrm{pH}$ 值来表征的特点, 提出关于 $C\left(\mathrm{H}^{+}\right)$的各组成作用的混合规则如下式:

$$
C\left(\mathrm{H}^{+}\right)=C\left(\mathrm{H}^{+}\right)_{1}^{\varphi_{1}} C\left(\mathrm{H}^{+}\right)_{2}^{\varphi_{2}}
$$

将公式(3), (4)，(7)和(8)代入公式(9), 并取负对数, 可得:

$$
p H=\varphi_{1} p H_{10}+\varphi_{2} p H_{20}+p A_{12} \varphi_{1} \varphi_{2}
$$

其中: $p A_{12}=0.5 \lg \left(A_{1,2} A_{2,1}\right)$, 定义为离子液体与水对溶液 体系整体酸碱性( $\mathrm{pH}$ 值)的交互作用因子.
离子液体 $[\mathrm{EMIM}] \mathrm{Ac}$ 和 $[\mathrm{HMIM}] \mathrm{Cl}$ 密度 $\rho$ 分别为 $1.1016 \mathrm{~g} / \mathrm{mL}$ 和 $1.0256 \mathrm{~g} / \mathrm{mL}^{[28]}$, 在水溶液中分别生成 $\mathrm{HAc}$ 和 $\mathrm{HCl}$, 这两种酸的 $p K_{\mathrm{a}}$ 值分别为 $4.75^{[29]}$ 和 $-8^{[30]}$. 根据公式(3), (4)和(10)拟合 $\mathrm{pH}$ 试纸的测定结果，离 子液体与水的极稀溶液的 $\mathrm{pH}$ 值 $p H_{10}$ 和 $p H_{20}$ 以及交互作 用因子 $p A_{12}$ 的值, 详见表 3 .

表 3 两种离子液体水溶液相关特性参数值

Table 3 Related parameters for the two ionic liquids aqueous solutions

\begin{tabular}{ccccccc}
\hline Ionic Liquid & $T_{\mathrm{d}} / \mathrm{K}$ & $p H_{10}$ & $p H_{20}$ & $p A_{12}$ & $\varepsilon$ & $\chi$ \\
\hline [EMIM]Ac & 495.75 & 7.10 & 6.80 & 2.22 & -0.055 & -17.02 \\
[HMIM]Cl & 537.95 & 7.80 & 6.15 & 0.871 & 0.06 & -2.68 \\
\hline
\end{tabular}

由上述模型对 $[\mathrm{EMIM}] \mathrm{Ac}$ 和 $[\mathrm{HMIM}] \mathrm{Cl}$ 两种水溶液 体系 $\mathrm{pH}$ 值的计算结果 $\left(p H_{\text {cal }}\right)$ 也分别列于表 1 和表 2 , 与 测定值进行比较, 并给 $\mathrm{pH}$ 测定值与计算值的相对误差 (RD). 相对误差计算式为:

$$
\mathrm{RD}=\frac{p H_{\mathrm{cal}}-p H_{\mathrm{exp}}}{p H_{\mathrm{exp}}}
$$

两种水溶液体系 $\mathrm{pH}$ 值计算结果分别示于图 2 和图 3(黑实线), 与测试结果进行比较. 由图可见离子液体水 溶液整体氢离子浓度模型计算结果与试纸测定结果能 够较好地符合.

由计算模型式(9)和式(10)可方便地计算任意浓度 离子液体水溶液的氢离子浓度, 再由离解反应平衡常数 $K_{\mathrm{a}}$ 的表达式(2)即可计算求得水溶液的酸浓度 $C(\mathrm{HD})$. 图 4 给出了两种离子液体水溶液中, 离子液体与水相互 作用产生的酸浓度计算值随溶液浓度变化的曲线, 其中 $[\mathrm{EMIM}] \mathrm{Ac}$ 和 $[\mathrm{HMIM}] \mathrm{Cl}$ 水溶液中生成的酸浓度分别记 为 $C(\mathrm{HAc})$ 和 $C(\mathrm{HCl})$. 由图可见, 对于[EMIM]Ac 水溶 液, 酸浓度小于 $10^{-9}$, 而对于 $[\mathrm{HMIM}] \mathrm{Cl}$ 水溶液, 酸浓度 小于 $10^{-20}$, 均小于水的自离解水平, 说明这两种离子液 体水溶液的水解程度很低.

\subsection{2 聚合膜中氢离子浓度计算模型}

本文在 $\mathrm{Guo}$ 等 ${ }^{[25]}$ 的研究基础上, 提出离子液体水溶 液在 $\mathrm{pH}$ 酸度计测量电极周围分层的概念, 论证使用 $\mathrm{pH}$ 酸度计和精密 $\mathrm{pH}$ 试纸测得实验数据的差异性.

Guo 等 ${ }^{[25]}$ 研究发现, 离子液体水溶液中有部分离子 液体会发生缔合惰化，惰化形态的离子液体分子不对水 分子活度产生影响，只有非惰化形态离子液体分子对水 分子活度产生作用, 可用溶液理论进行分析, 并给出了 $[\mathrm{EMIM}] \mathrm{Ac}$ 和 $[\mathrm{HMIM}] \mathrm{Cl}$ 在水溶液中缔合惰化因子 $\beta$ 的 计算公式.

在聚合膜中，由于惰化离子液体占主导，非惰化离 子液体部分的影响力有限, 只有当混合体系中水的份额 不断增加，使得惰化离子液体浓度降低到一定程度时， 


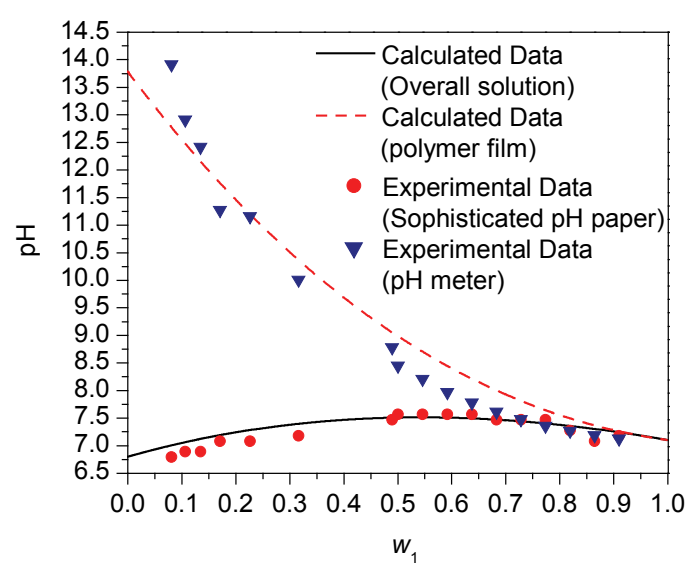

图 2 [EMIM] Ac 水溶液 $\mathrm{pH}$ 计算值与拟合值

Figure $2 \mathrm{pH}$ Value experimental and calculated data for [EMIM]Ac aqueous solution

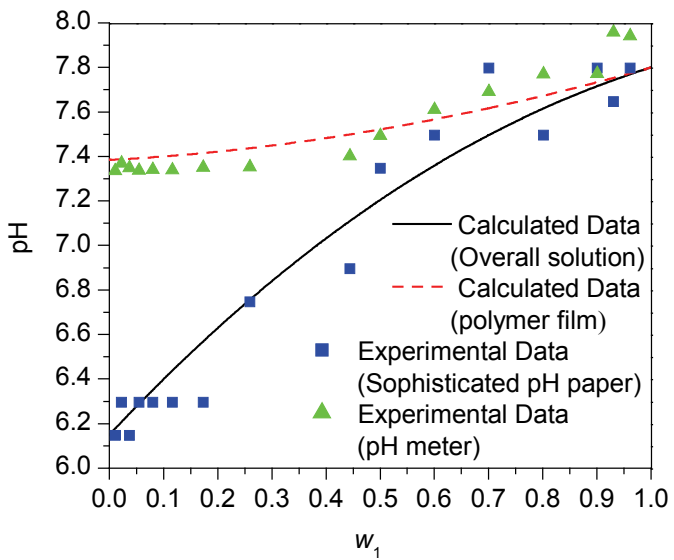

图 3 [HMIM $] \mathrm{Cl}$ 水溶液 $\mathrm{pH}$ 计算值与拟合值

Figure $3 \mathrm{pH}$ Value experimental and calculated data for [HMIM]Cl aqueous solution

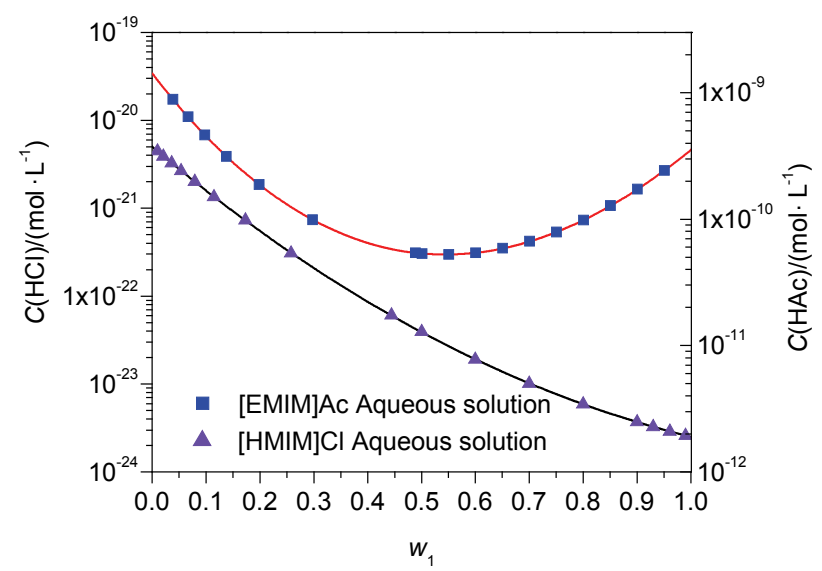

图 4 离子液体水溶液中酸浓度随浓度变化

Figure 4 Acid concentration for ionic liquids aqueous solutions (HAc for [EMIM] Ac aqueous solution; $\mathrm{HCl}$ for [HMIM]Cl aqueous solution)

非惰化离子液体的影响力才成为主导, 使得离子液体聚 合膜弱化为溶体, 使得 $\mathrm{pH}$ 酸度计和精密 $\mathrm{pH}$ 试纸测定的 $\mathrm{pH}$ 值趋于一致. 在图 1 所示的聚合膜 $\mathrm{B}$ 相和非惰化离
子液体水溶液 $\mathrm{C}$ 相中水的逸度值分别计为 $f_{1} \mathrm{~B}$ 和 $f_{1}^{\mathrm{C}}$, 可 由水在两相中的体积分数 $\varphi_{1}{ }^{\prime}$ 和 $\varphi_{1}$ 以及对应的亨利系数 $H_{1,2}$ '和活度系数 $\gamma_{1}$ 来表示:

$$
\begin{aligned}
& f_{1}^{B}=H_{1,2}{ }^{\prime} \varphi_{1}{ }^{\prime} \\
& f_{1}^{\mathrm{C}}=\gamma_{1} \varphi_{1}
\end{aligned}
$$

这里上标 ' 表示在聚合膜相. 当两相中水份达到 平衡时, $f_{1}^{\mathrm{B}}$ 和 $f_{1}^{\mathrm{C}}$ 相等, 可获得两相中水份额平衡系数 $K$ :

$$
K=\frac{\varphi_{1}^{\prime}}{\varphi_{1}}=\frac{\gamma_{1}}{H_{1,2}^{\prime}}
$$

在离子液体组分中, 惰化和非惰化两种类型分子所 占份额分别为 $\beta$ 和 $1-\beta, \beta$ 可由文献 ${ }^{[25]}$ 提供的公式计算:

$$
\beta=0.98 \exp \left(-0.33\left(\frac{T-273.15}{T_{\mathrm{d}}-T}\right)^{3}\right)
$$

式中 $T$ 为实验温度, $T_{\mathrm{d}}$ 为离子液体分解温度.

聚合膜对水的溶解度由惰化和非惰化离子液体共 同决定，因此在聚合膜中溶解水的亨利系数可由下式确 定:

$$
\ln \left(H_{1,2}{ }^{\prime}\right)=(1-\beta) \varphi_{2} \ln \left(\gamma_{1}^{\prime}\right)+\beta \varphi_{2} \ln \left(H_{1,2}{ }^{(0)}\right)
$$

式中, $H_{1,2}{ }^{(0)}$ 为惰化离子液体中水分压为零时的亨利系 数.

根据 Flory-Huggins 理论，在含有大分子的溶液中， 小分子的活度系数 $\gamma$ 由溶液中分子的相互作用参数 $\chi$ 和分子所含链接数 $r$, 以及大分子的体积分数决定. 对 于非惰化离子液体水溶液相, 水的活度系数 $\gamma_{1}$ 可根据 公式(17)计算:

$$
\ln \left(\gamma_{1}\right)=\left(1-\frac{1}{r}\right) \varphi_{2}+\chi \varphi_{2}^{2}
$$

在聚合膜相中, 因为水的体积分数极小, $\varphi_{1} \approx 0$, $\varphi_{2} \approx 1$, 因此, 在聚合膜相中水的活度系数可采用下式 计算:

$$
\ln \left(\gamma_{1}^{\prime}\right)=\left(1-\frac{1}{r}\right)+\chi
$$

将式(14)两边取自然对数, 同时将式(16), (17)和 (18)代入得:

$$
\begin{aligned}
\ln (K) & =\left(1-\frac{1}{r}\right) \varphi_{2}+\chi \varphi_{2}{ }^{2}- \\
& {\left[(1-\beta) \varphi_{2}\left(1-\frac{1}{r}+\chi\right)+\beta \varphi_{2} \ln \left(H_{1,2}{ }^{(0)}\right)\right] }
\end{aligned}
$$

引入由式(19)中各待定系数定义的一个常量影响因 子 $\varepsilon$ : 


$$
\varepsilon=\frac{1-\frac{1}{r}-(1-\beta)\left(1-\frac{1}{r}+\chi\right)-\beta \ln \left(H_{1,2}{ }^{(0)}\right)}{\chi}
$$

则式(19)可简化为:

$$
\ln (K)=\left(\varepsilon+\varphi_{2}\right) \chi \varphi_{2}
$$

式中参数 $\varepsilon$ 和 $\chi$ 可通过拟合实验数据获得.

由于氢离子浓度与水的浓度(或容积分数)成比例, 因此聚合膜和水溶液两相的氢离子浓度之比应等于水 的容积分数之比, 即等于两相水组分的平衡系数 $K$, 即 有:

$$
K=\frac{10^{-p H^{\prime}}}{10^{-p H}}
$$

由式(21)和(22), 可得到离子液体聚合膜 $\mathrm{pH}$ 值与水 溶液体系 $\mathrm{pH}$ 值的差值(即 $\mathrm{pH}$ 酸度计测得值与 $\mathrm{pH}$ 试纸 测得值之差):

$$
p H^{\prime}-p H=0.4343\left(\varepsilon+\varphi_{2}\right) \chi \varphi_{2}
$$

式中 $p H$ 可由式(10)确定. 用式(23)分别对 $\mathrm{pH}$ 酸度计测 得的 $[\mathrm{EMIM}] \mathrm{Ac}$ 和 $[\mathrm{HMIM}] \mathrm{Cl}$ 水溶液 $\mathrm{pH}$ 值进行拟合可得 到两种水溶液的聚合膜和水溶液相的作用参数 $\varepsilon$ 和 $\chi$ 的 值，具体见表 3.

根据以上建立的数学模型, 式(10)和(23), 可得到 对应于 $\mathrm{pH}$ 酸度计的计算值 $\left(p H_{c a l}\right)$. 在表 1 和表 2 中也 分别列出[EMIM] $\mathrm{Ac}$ 和 [HMIM] $\mathrm{Cl}$ 聚合膜的 $\mathrm{pH}$ 值计算结 果, 并与 $\mathrm{pH}$ 酸度计测得的值进行比较, 其平均相对误 差分别小于 $5 \%$ 和 $2 \%$. 对两种离子液体聚合膜 $\mathrm{pH}$ 值计 算结果也分别在图 2 和图 3 中给出, 由虚线所示, 由图 可见模型计算结果与测定结果能够很好地符合.

由表 1 和表 2 可以看出, 当水的质量分数为 $0.5 \sim$ 0.8 时, [EMIM] Ac 水溶液使用 $\mathrm{pH}$ 酸度计测得的实验值 与拟合值误差较大，在水质量分数为 0.49 和 0.5 之间, 实验值略有突降, 这是 $\mathrm{pH}$ 酸度计自身测量误差造成. 当水质量分数小于 0.2 时, [HMIM]Cl 水溶液 $\mathrm{pH}$ 试纸测 试值与拟合值相对误差较大, 但在 0.2 范围内, 而精密 试纸的分辨精度为 $0.2 \sim 0.3$, 测试值精度由精密试纸读 取精度所限制.

\section{3 结论}

本文采用 $\mathrm{pH}$ 酸度计和精密 $\mathrm{pH}$ 试纸两种方法, 对 1-乙基-3-甲基咪唑醋酸盐([EMIM]Ac)和 1-己基-3-甲基 咪唑氯盐([HMIM]Cl)离子液体水溶液的 $\mathrm{pH}$ 值进行测试 和定量分析. 由研究结果可获得如下几点结论：

(1)使用 $\mathrm{pH}$ 酸度计和精密 $\mathrm{pH}$ 试纸分别测得的离子 液体水溶液 $\mathrm{pH}$ 值有较大差异. 该差异是由于离子液体水混合体系中局部形态与整体特性不同造成的. 精密 $\mathrm{pH}$ 试纸测定的是溶液总体酸碱特性, 而 $\mathrm{pH}$ 酸度计测定 的是富集于 $\mathrm{pH}$ 酸度计测量电极周围惰化离子液体聚合
膜中的氢离子浓度.

(2)精密试纸测定的 $\mathrm{pH}$ 值可用于表征离子液体水溶 液整体酸碱特性. 提出了稀溶液氢离子浓度计算模型以 及溶液组分作用模型和混合规则，建立了离子液体水溶 液整体 $\mathrm{pH}$ 值的预测方法，成功拟合了 $\mathrm{pH}$ 精密试纸测定 值，并计算获得了两种离子液体水溶液的酸浓度.

(3)根据离子液体在水溶液中形成惰化聚合膜的假 设, 提出了分别表征聚合膜水溶解度和离子液体水溶液 水活度系数的溶液理论模型, 并由溶液相平衡理论建立 了聚合膜中氢离子浓度与水溶液中氢离子浓度差别的 计算方法，成功拟合了 $\mathrm{pH}$ 酸度计测定值.

研究结果为离子液体水溶液体系的 $\mathrm{pH}$ 值和酸碱度 分析提供了实验依据和预测理论模型.

\section{4 实验部分}

\section{1 实验试剂}

1-乙基-3-甲基咪唑醋酸盐 ([EMIM]Ac): 纯度为 $98 \%$, 化学式为 $\mathrm{C}_{8} \mathrm{H}_{14} \mathrm{~N}_{2} \mathrm{O}_{2}$, 分子量 170.21 , 室温下黏度 为 $83.51 \mathrm{mPa} \cdot \mathrm{s}^{[31]} ; 1$-已基-3-甲基咪唑氯盐([HMIM]Cl): 纯度为 $98 \%$, 化学式为 $\mathrm{C}_{10} \mathrm{H}_{19} \mathrm{~N}_{2} \mathrm{Cl}$, 分子量为 202.72 , 室温下黏度大于 $1800 \mathrm{mPa} \bullet \mathrm{s}^{[32]}$; 两种离子液体均由中国 科学院兰州物理化学研究所提供. 去离子水为实验室自 制，其电导率小于 $1 \mu \mathrm{S} \cdot \mathrm{cm}^{-1}$.

\section{2 实验设备}

微量水分滴定仪, SFY-3A 型, 淄博海分仪器厂, 测 量值为 $10 \mu \mathrm{g} \sim 1 \mathrm{mg}$ 时, 精度为 $\pm 3 \mu \mathrm{g}$, 测量值大于 1 $\mathrm{mg}$ 时, 精度为 $\pm 0.5 \%$, 用于测定离子液体初始存留含 水量. $\mathrm{pH}$ 酸度计, PHSJ-4A 型, 上海精密科学仪器有限 公司, 精度为 $\pm 0.02 \mathrm{pH}$. 精密 $\mathrm{pH}$ 试纸, 德国 MACHEREY-NAGEL，选用三种不同型号：REF90420, RE90417 和 REF90415, 精度 $\pm 0.2 \sim 0.3 \mathrm{pH}$. 分析天平, CP225D 型，德国 SARTORIUS，精度 $\pm 0.01 \mathrm{mg}$ ，用于称 量离子液体和水. 空气干燥机, WED-001, 广州维通工 业气体技术有限公司, 用于提供干燥空气氛围.

\section{3 实验过程}

首先在高纯氮密闭环境中用微量水分滴定仪对离 子液体中的初始水含量进行测定, 实验测试系统如支持 信息图 S1 所示. 测得[EMIM]Ac 和[HMIM]Cl 的初始含 水量分别为 $8700 \pm 500 \mathrm{ppm}$ 和 $6100 \pm 500 \mathrm{ppm}$. 利用精 密天平称重, 在一组 $10 \mathrm{~mL}$ 量筒中配置约 $5 \mathrm{~mL}$ 的不同 质量分数的离子液体水溶液测试样品, 并密封保存备 用. 在 $0.01 \sim 0.96$ 范围内, 每种离子液体分别配制 16 个 不同水质量分数的水溶液样品. 依据称重质量并综合考 虑离子液体的初始存留水含量计算获得的水质量百分 数不确定度小于 $0.1 \%$.

离子液体水溶液样品配置好之后, 在干燥空气的保 护下使用 $\mathrm{pH}$ 酸度计与精密 $\mathrm{pH}$ 试纸分别对[EMIM]Ac 
和 $[\mathrm{HMIM}] \mathrm{Cl}$ 两种离子液体水溶液各 16 种样品的 $\mathrm{pH}$ 值 进行测定，实验测试系统见支持信息图 S2 所示.

测试过程在室温 $\left(25{ }^{\circ} \mathrm{C}\right)$ 和 $1 \mathrm{~atm}$ 的室内环境条件下 进行. 使用 $\mathrm{pH}$ 酸度计进行 $\mathrm{pH}$ 值测定时分三步，第一, 使用标准缓冲液标定 $\mathrm{pH}$ 酸度计, 然后用去离子水冲洗 酸度计测量球泡，并用吸水纸吸干测量球泡周围的水; 第二, 将盛有离子液体水溶液的量筒放入留有窄开口的 保护容器中, 干燥空气不断从容器底部通入, 防止离子 液体水溶液吸收空气中的水份造成离子液体浓度偏差; 第三, 将测量电极的测量球泡浸没入被测溶液中, 开始 $\mathrm{pH}$ 值的测量, 约 $20 \mathrm{~min}, \mathrm{pH}$ 数值稳定后, 记录 $\mathrm{pH}$ 值, 并用大量去离子水清洗测量电极, 吸干测量球泡周围的 水分, 进行下一个试样 $\mathrm{pH}$ 值的测定. 使用精密 $\mathrm{pH}$ 试纸 时, 将试纸放在干燥的玻璃皿中, 使用干净的玻璃棒沾 取离子液体均匀涂抹在精密 $\mathrm{pH}$ 试纸表面, 使试纸的测 试区和对照区全部浸润在离子液体水溶液中, 约 $3 \mathrm{~s}$ 后, 读取与测试区颜色最相近的对照区读数, 即溶液的 $\mathrm{pH}$ 值, 每个试样使用精密 $\mathrm{pH}$ 试纸进行三次测量, 取平均 值.

\section{References}

[1] Gao, X.; Fan, J.; Wang, X. L.; Zhang, Y. S. Acta Chim. Sinica 2013, 71(10), 1411. (高霞, 樊静, 王小龙, 张艳树, 化学学报, 2013, 71(10), 1411.)

[2] Huang, B.; Li, Z.; Wang, Y.; Zhang, Y.; Fang, Y. Acta Chim. Sinica 2008, 66 (15), 1837. (黄宝华, 黎子进, 汪艳飞, 张炟, 方岩雄, 化 学学报, 2008, 66(15), 1837.)

[3] Huo, C. D.; Wang, C. Chin. J. Org. Chem. 2013, 33, 2108. (霍聪德, 王程, 有机化学, 2013, 33, 2108.)

[4] Zhang, P.; Du, J.; Yang, F.; Zou, G.; Tang, J. Chin. J. Chem. 2005, 23(5), 581 .

[5] Yokozeki, A.; Shiflett, M. B. Appl. Energy 2007, 84(12), 1258.

[6] Shiflett, M. B.; Yokozeki, A. US 20060197053, Al 2006 [Chem. Abstr. 2006, 2597199 A1].

[7] Wei, Z.; Wu, X. H.; Zheng, D. X.; Wang, J. Z.; Dong, L. J. Beijing Univ. Chem. Technol. (Nat. Sci. Ed.) 2010, 37(01), 9. (魏治, 武向 红, 郑丹星, 王建召, 董丽, 北京化工大学学报 (自然科学版), 2010, 37(01), 9.)

[8] Tian, T.; Zheng, D. X.; Wu, X. H.; Jiang, Y. R. J. Beijing Univ. Chem. Technol. (Nat. Sci. Ed.) 2008, 35(03), 27. (田涛，郑丹星，武 向红, 蒋翼然, 北京化工大学学报 (自然科学版), 2008, 35(03), 27.)
[9] Dong, L.; Zheng, D. X.; Wu, X. H.; Li, F.; Wei, Z. J. Eng. Thermophys. 2009, 30(08), 1271. (董丽，郑丹星，武向红，李枫，魏治，工 程热物理学报, 2009, 30(08), 1271.)

[10] Guan, T. T.; Sun, L.; Huangfu, L. X.; Guo, K. H. Chinese J. Low Temp. Phys. 2011, 33(03), 194. (关婷婷, 孙立, 皇甫立霞, 郭开 华, 低温物理学报, 2011, 33(03), 194.)

[11] Sun, L.; Guo, K. H.; Huangfu, L. X. Chinese J. Low Temp. Phys. 2011, 33(05), 381. (孙立, 郭开华, 皇甫立霞, 低温物理学报, 2011, 33(05), 381.)

[12] Yang, T.; Bi, Y.; Guo, K. H. CIESC J. 2012, 63(10), 3152. (阳涛, 毕峑, 郭开华, 化工学报, 2012, 63(10), 3152.)

[13] Yuan, X.; Zhang, S.; Liu, J.; Lu, X. Fluid Phase Equilib. 2007, $257(2), 195$.

[14] Pan, S. F.; Hu, G. X.; Lv, Y.; Zou, J. W.; Yu, Q. S. Acta Phys.-Chim. Sin. 2010, 26(9), 2494. (潘善飞, 胡桂香，吕杨，邹建 卫, 俞庆森, 物理化学学报, 2010, 26(9), 2494.)

[15] Liang, R.; Yang, M. R.; Zhou, Q. X. Acta Phys.-Chim. Sin. 2010 26(6)，1468. (梁芯，杨美荣，周庆祥，物理化学学报, 2010, 26(6), 1468.)

[16] Aggarwal, V. K.; Emme, I.; Mereu, A. Chem. Commun. 2002, 1612.

[17] Seddon, K. R. J. Chem. Technol. Biot. 1997, 68(4), 351.

[18] Fei, Z.; Zhao, D.; Geldbach, T. J.; Scopelliti, R.; Dyson, P. J. Chem -Eur. J. 2004, 10(19), 4886.

[19] Thomazeau, C.; Olivier-Bourbigou, H.; Magna, L.; Luts, S.; Gilbert, B. J. Am. Chem. Soc. 2003, 125(18), 5264.

[20] Yang, Y.; Kou, Y. Chem. Commun. 2004, 226.

[21] Chen, X. W.; Song, H. B.; Chen, P.; Wang, F. R.; Qian, Y.; Li, X. H. Acta Chim. Sinica. 2012, 70, 770. (陈学伟，宋红兵，陈鹏，王芙 蓉, 钱宇, 李雪辉, 化学学报, 2012, 70, 770.)

[22] Ober, C. A.; Gupta, R. B. Ind. Eng. Chem. Res. 2012, 51(6), 2524.

[23] Guo, K.; Bi, Y.; Sun, L.; Su, H.; Hungpu, L. J. Chem. Eng. Data 2012, 57(8), 2243.

[24] Su, H.; Guo, K. H.; Huangfu, L. X.; Sun, L. J. Refrig. 2013, 34(3), 24. (粟航，郭开华，皇甫立霞，孙立，制冷学报, 2013, 34(3), 24.)

[25] Guo, K.; Bi, Y.; Sun, L.; Su, H.; Hungpu, L. J. Chem. Eng. Data 2012, 57(8), 2243.

[26] Singh, T.; Kumar, A. Colloids Surf. A 2008, 318(1), 263

[27] Fu, S. Z.; Chen, Q. D.; Shen, X. H. Acta Phys.-Chim. Sin. 2011, 27(8), 1913. (付素珍, 陈庆德, 沈兴海, 物理化学学报, 2011 27(8), 1913.)

[28] Sun, L.; Guo, K. H.; Huangfu, L. X. Chin. J. Low Temp. Phys. 2011, 33(6)，467. (孙立, 郭开华, 皇甫立霞, 低温物理学报, 2011, 33(6), 467.)

[29] Long, Y. H.; Xiang, M. L.; Gao, Y. H. J. Chongqing Institute Technol. 2001，15(05), 98. (龙彦辉，向明礼，高彦荷，重庆工学院学 报, 2001, 15(05), 98.)

[30] http://www.chem.wisc.edu/areas/reich/pkatable/index.htm, 2013.7.22[Z]

[31] Hou, H.; Huang, Y. R.; Wang, S. Z.; Bai, B. F. Acta Phys.-Chim. Sin. 2011, 27(11), 2512. (侯海云, 黄银蓉, 王升泽, 白博峰, 物理 化学学报, 2011, 27(11), 2512.)

[32] Ning, H.; Hou, M.; Mei, Q.; Liu, Y.; Yang, D.; Han, B. Sci. China Chem. 2012, 55(08), 1509. 